\title{
Investigating microstructures in 3D: Advances in modern instruments and analytics \\ RICH TAYLOR
}

Carl Zeiss Microscopy Ltd

Presenting Author: richard.taylor@zeiss.com

The proliferation of modern techniques available for petrologists has resulted in an explosion of detailed information on mineral compositions and processes over the last decade. One area of research that is undergoing dramatic advances is the nondestructive interrogation of samples in three dimensions through X-ray microscopy (XRM). Techniques such as ZEISS Versa, Context and Ultra XRM can be performed at a variety of scales and resolutions, resulting in micro-to-nano scale information on geological samples. Such techniques can be correlated with each other i.e. expanding nanoscale resolution to a large sample, internal calibration of X-ray intensity to identify mineral assemblages, or even correlation with other techniques such as electron microscopy (EM). X-ray techniques are also particularly adaptable to digital resolution enhancements through software processes such as machine learning algorithms that can dramatically speed up routine tasks.

Collecting 3D information for petrological investigations can often require ground truthing of mineralogical and compositional interpretations. The more developed the 3D microscopy becomes, the more we are increasingly interested in features that are deeply buried within our samples. This means the corresponding techniques for excavating a region of interest also need to advance in both speed and accuracy.

The ZEISS Crossbeam-Laser (XBL) system provides a unique capability of rapidly excavating to a point of interest within a $3 \mathrm{D}$ sample volume. The XBL is already seeing use in material sciences, with a standard XB chamber with FIB and EM, and a correlated femtosecond laser chamber for rapid material removal. Sample data collected through XRM can be correlated to the XBL stage so that any internal features located by XRM have their coordinates automatically available in three dimensions. The femtosecond laser can excavate to a region of interest (RoI) within the sample within seconds or minutes, dramatically reducing preparation time compared to standard FIB/PFIB. The laser cut surface can be used for analysis techniques such as energy dispersive spectroscopy (EDS) and electron backscatter diffraction (EBSD), even prior to final polishing with the focussed ion beam (FIB). 